\title{
Allelic variation contributes to bacterial host specificity
}

\author{
Min Yue ${ }^{1}$, Xiangan Han ${ }^{1, \dagger}$, Leon De Masi ${ }^{1}$, Chunhong Zhu ${ }^{1, \dagger}$, Xun Ma ${ }^{1, \dagger}$, Junjie Zhang ${ }^{1, \dagger}$, Renwei Wu ${ }^{1, \dagger}$, \\ Robert Schmieder ${ }^{2, \dagger}$, Radhey S. Kaushik ${ }^{3,4}$, George P. Fraser ${ }^{5}$, Shaohua Zhao ${ }^{6}$, Patrick F. McDermott ${ }^{6}$, \\ François-Xavier Weill ${ }^{7}$, Jacques G. Mainil ${ }^{8}$, Cesar Arze ${ }^{9}$, W. Florian Fricke9, ${ }^{\dagger}$, Robert A. Edwards ${ }^{2,10}$, \\ Dustin Brisson ${ }^{11}$, Nancy R. Zhang ${ }^{12}$, Shelley C. Rankin ${ }^{1} \&$ Dieter M. Schifferli ${ }^{1}$
}

Understanding the molecular parameters that regulate cross-species transmission and host adaptation of potential pathogens is crucial to control emerging infectious disease. Although microbial pathotype diversity is conventionally associated with gene gain or loss, the role of pathoadaptive nonsynonymous single-nucleotide polymorphisms (nsSNPs) has not been systematically evaluated. Here, our genome-wide analysis of core genes within Salmonella enterica serovar Typhimurium genomes reveals a high degree of allelic variation in surface-exposed molecules, including adhesins that promote host colonization. Subsequent multinomial logistic regression, MultiPhen and Random Forest analyses of known/suspected adhesins from 580 independent Typhimurium isolates identifies distinct host-specific nsSNP signatures. Moreover, population and functional analyses of host-associated nsSNPs for $\mathrm{FimH}$, the type 1 fimbrial adhesin, highlights the role of key allelic residues in host-specific adherence in vitro. Together, our data provide the first concrete evidence that functional differences between allelic variants of bacterial proteins likely contribute to pathoadaption to diverse hosts.

\footnotetext{
${ }^{1}$ Department of Pathobiology, University of Pennsylvania School of Veterinary Medicine, 3800 Spruce St, Philadelphia, Pennsylvania 19104, USA. ${ }^{2}$ Department of Computer Science, College of Sciences, San Diego State University, 5500 Campanile Drive, PS 106, San Diego, California 92182, USA. ${ }^{3}$ Department of Veterinary and Biomedical Sciences, South Dakota State University, Brookings, South Dakota 57007, USA. ${ }^{4}$ Department of Biology and Microbiology, Box-2140D, South Dakota State University, Brookings, South Dakota 57007, USA. ${ }^{5}$ Pennsylvania Department of Health, Bureau of Laboratories, 110 Pickering Way, Exton, Pennsylvania 19341, USA. ${ }^{6}$ Division of Animal and Food Microbiology, Center for Veterinary Medicine, US FDA, Office of Research, 8401 Muirkirk Road, Laurel, Maryland 20708, USA. ${ }^{7}$ Institut Pasteur, Unité des Bactéries Pathogènes Entériques, 28 rue du Docteur Roux, 75724 Paris Cedex 15, France. 8 Bacteriology, Department of Infectious Diseases, Faculty of Veterinary Medicine and Institute for Fundamental and Applied Research in Animal Health (FARAH), Campus du Sart Tilman, Bât. B43a, University of Liège, 4000 Liège, Belgium. ${ }^{9}$ Institute for Genome Sciences, Department for Microbiology and Immunology, University of Maryland School of Medicine, Baltimore, 801W Baltimore St, Maryland 21201, USA. ${ }^{10}$ Mathematics and Computer Science Division, Argonne National Laboratory, 9700S. Cass Avenue, Argonne, Illinois 60439, USA. ${ }^{11}$ Department of Biology, School of Art and Science, University of Pennsylvania, 209 Leidy Laboratories, 433S. University Avenue, Philadelphia, Pennsylvania 19104, USA. ${ }^{12}$ Department of Statistics, Wharton School, University of Pennsylvania, 3730 Walnut Street, Philadelphia, Pennsylvania 19104, USA. † Present addresses: Shanghai Veterinary Research Institute, Chinese Academy of Agricultural Sciences (CAAS), Ziyue Road \#518, Minhang District, Shanghai 200241, PR China (X.H.); Jiangsu Institute of Poultry Science, Chinese Academy of Agricultural Sciences, Yangzhou, 225125 Jiangsu, PR China (C.Z.); Department of Veterinary Medicine, College of Animal Science and Technology, Shihezi University, 280N 4th Road, Shihezi 832003, PR China (X.M.); College of Food and Biological Engineering, Zhengzhou University of Light Industry, 5 Dongfeng Road, Jinshui District, Zhengzhou 450002, PR China (J.Z.); College of Food Science and Technology, \#1 Shizhisan Road, Wuhan 430070, PR China (R.W.); Illumina, Inc., 5200 Illumina Way, San Diego, California 92122, USA (R.S.); Department of Biological Chemistry and Nutrition, University of Hohenheim, Schloss Hohenheim 1, 70599 Stuttgart, Germany (W.F.F.). Correspondence and requests for materials should be addressed to D.M.S. (email: dmschiff@vet.upenn.edu).
} 
E volutionary events that modulate interactions between a pathogen and its host have a critical impact on interspecies transmission and adaptation, and thus on host range and pathogenesis ${ }^{1}$. For example, although most avian influenza viruses remain restricted to birds, some, such as $\mathrm{H} 5 \mathrm{~N} 1$ and H7N9, cause serious infections in humans ${ }^{2}$. The ability of influenza viruses to cross host barriers is determined by the amino-acid sequence of its haemagglutinin protein, a lectin that recognizes sialylated glycan receptors on the apical surface of host cells. Even small changes of a few amino acids in the haemagglutinin protein are sufficient to convert receptor specificity from avian to human ${ }^{1,2}$. The exact mechanisms for host tropism and adaptation of bacterial pathogens such as Salmonella remain elusive, and what is known has been primarily revealed by either gene-centric functional investigations ${ }^{3-5}$ or by genomic studies 6,7 . Moreover, the biological relevance of most host-specific associations identified in genome-wide studies remains untested ${ }^{8-10}$.

The $\sim 1,500$ closely related but distinct Salmonella enterica subsp. enterica serovars, determined by lipopolysaccharides and flagellar antigens, can be divided into three groups based on epidemiological host prevalence. Many S. enterica serovars such as $S$. enterica serovar Typhimurium ( $S$. Typhimurium) are restricted to the intestine, and cause limited clinical or subclinical enteric infections in a variety of unrelated hosts. In contrast, several $S$. enterica serovars that are particularly well adapted to their host, including Typhi in humans and Gallinarum in poultry, are more invasive, and result in a systemic infection that can be lethal if not treated promptly with antibiotics. However, epidemiological evidence supports various levels of host adaptation even among strains of broad host range serovars. For example, whereas most strains of $S$. Typhimurium cause a typhoid-like disease in susceptible mice, particular phage types such as DT2 or DT99 can cause systemic infections in pigeons ${ }^{11}$ and the multi-locus sequence type ST313 causes systemic infection in humans and chickens ${ }^{12,13}$. Thus, it appears that both inter- and intra-serovar variation have a role in host range and disease severity. Here we undertook a genome-wide search to identify genomic sequences that contribute to host adaptation and surprisingly found that allelic variants of shared surface adhesion molecules correlated most strongly with host specificity. Most importantly, functional analysis of identified variants of the FimH adhesin confirmed their biological relevance in modulating host-specific binding that can contribute to host-adaptation and ultimately to the Salmonella strain pathotype.

\section{Results}

Detection of genome-host associations in $S$. Typhimurium. To determine what genomic changes contribute to host association in Salmonella, we focused on S. Typhimurium, a broad-host range serovar for which the molecular basis for host preferences remains essentially unknown. A comparative analysis of the 3,192 core genes from 12 available complete genomes of S. Typhimurium (Supplementary Fig. 1a) detected a relative and preferential accumulation of single-nucleotide polymorphisms (SNPs) among the annotated genes for membrane-associated surface and exported proteins (Fig. 1a). Most SNPs (69\%) were nonsynonymous (nsSNPs). Noticeably, nsSNPs in the core genomes associated with specific hosts, as shown in a heat map of nsSNPs (Supplementary Fig. 1b), suggesting that host preferences of individual Typhimurium strains involves unique combinations of cell surface and exported allelic proteins. In contrast, no hostspecific associations were identified in the 2,312 partially shared genes, which were frequently associated with loci carrying phage DNA (Supplementary Fig. 1c and Supplementary Table 1), the
1,207 unique genes, which were mostly mobile elements (Supplementary Fig. 1c,d), the 19 different plasmids (Supplementary Data 1), the few detected genomic rearrangements (Supplementary Fig. 2), or the shared pseudogenes (Supplementary Fig. 3). As the most distinctive genomic property of $S$. Typhimurium's association to diverse hosts was its allelic variants of surface or exported proteins, we further investigated the potential role of a representative set of these proteins in hostpathogen interactions that may contribute to host adaptation.

Distinct S. Typhimurium adhesin SNPs are host specific. Adhesive proteins or ligands on bacteria are likely to have an essential role in initiating host-pathogen interactions that contribute to host adaptation. Therefore, we undertook a populationscale analysis of 580 Typhimurium strains and focused on 12 known or predicted fimbrial adhesins and 3 outer membrane proteins suspected to have binding properties. Sequence data were collected from 198 available genomes (Supplementary Data 2) and from targeted sequencing of 382 independent isolates (Supplementary Data 3$)^{14}$. We first investigated the degree of variability for the 15 genes, and found that fim $\mathrm{H}$ encoded the largest number of different alleles (Supplementary Table 2). Tajima's $D$ tests for non-neutral evolution suggested that all 15 genes underwent positive selection. SNPs from these 15 genes were further analysed for host association using Random Forest (RF), multinomial logistic regression and MultiPhen analyses (Supplementary Data 4-6) ${ }^{15,16}$. A total of 182 host-associated mutations were detected by at least one method, 82 by two methods and 32 by all three methods (Supplementary Fig. 5). Of the 32 consistently identified host-associated mutations, half were nsSNPs, two of which were related to the absence or presence of a full-length open-reading frame (Supplementary Table 3). Moreover, a 3D scaling plot from the RF analysis that separated subpopulations of isolates from the same host clearly revealed host-specific DNA signatures (Fig. $1 \mathrm{~b}$ and Supplementary Fig. 4). Collectively, these in silico data strongly suggest that allelic variation in known or predicted bacterial adhesins of a broad-host range serovar is associated with hostspecificity.

\section{A S. Typhimurium FimH residue shapes host-specific adhesion} The next goal was to evaluate whether a detected host-associated allelic variant effectively alters bacterial binding in a host-specific manner. Among the 15 genes studied in S. Typhimurium, we found that $f \mathrm{mH}$ encoded the greatest number of predicted alleles, with 17 identified variants, most of which were expressed in only one to three strains. However, two alleles were present in over 30 isolates. Although fimH1 was a broad adaptive allele, it most frequently associated with human isolates, whereas the second most common allele, fim $\mathrm{H}$, was most frequently associated with isolates of bovine origin (Fig. 2a; $P<0.0009$, Fisher's exact test). These two alleles differ by only one amino acid; with a valine for FimH1 and an alanine for FimH7 at position 223. To determine the potential biological function of this residue switch, we tested the binding property of recombinant E. coli expressing isogenic $S$. Typhimurium type 1 fimbriae with either one of the two allelic FimH proteins or no FimH as control. Using three human and four bovine intestinal epithelial cells, the affinity of $S$. Typhimurium FimH7 was greater than that of FimH1 for all bovine cells (Fig. 2b). Although FimH1 appeared to preferentially bind to some human cells, the difference was not statistically significant. Nevertheless, these results indicated that the alanine at position 223 in FimH7 has a role in bovine-specific adhesion. Like FimH1, all 48 available genome sequences of the human-restricted $S$. Typhi encode a valine at position 223 . Thus, to determine whether this 
a

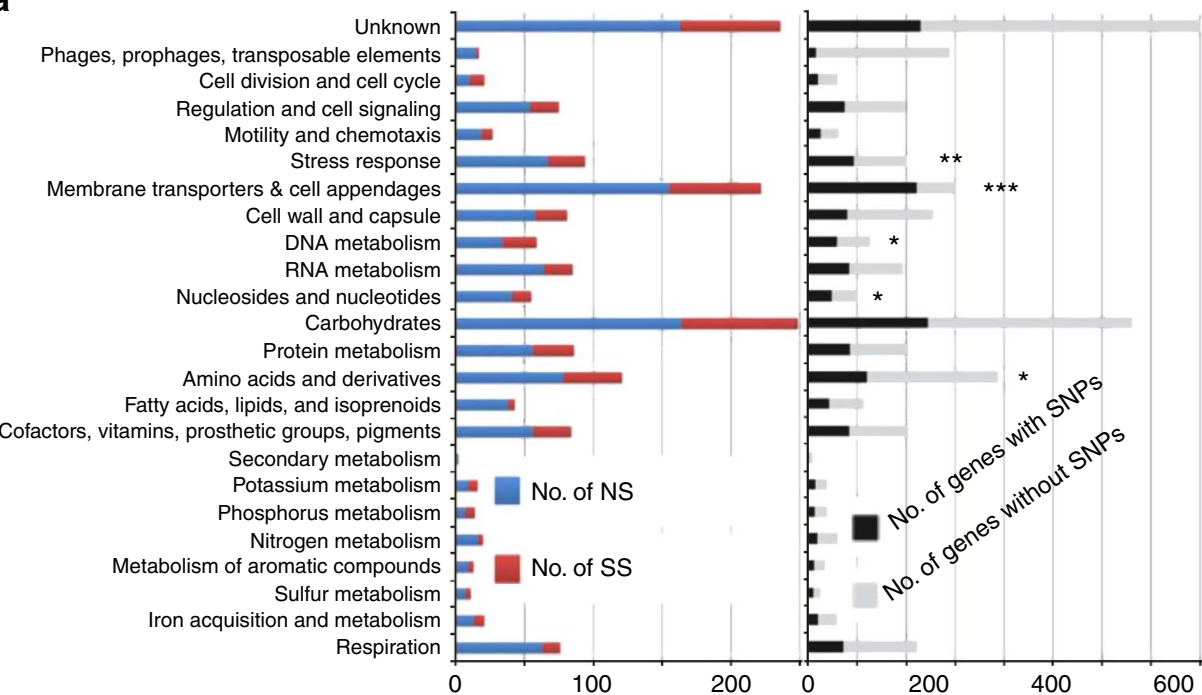

b

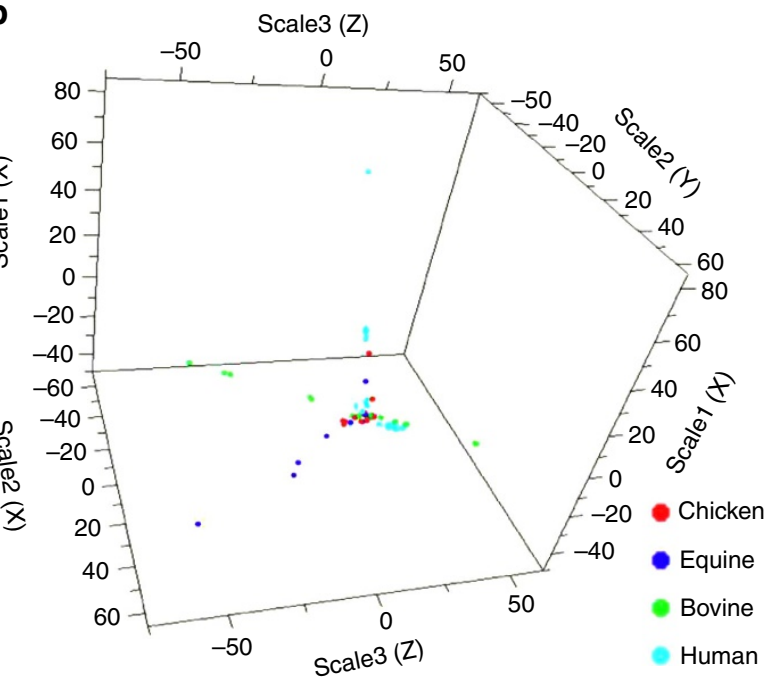

Figure 1 | Comparative analysis and host origin association for S. Typhimurium genomes. (a) Functional distribution of core genes in 12 isolates; at left total numbers of SNPs with proportions of nonsynonymous substitutions (NS, blue) and synonymous substitutions (SS, red), and at right, the number of genes with (black) and without SNPs (grey). P-values for associations of sets of genes with SNPs and a given functional category (right-tailed Fisher exact test): ${ }^{\star} P<0.05$; ${ }^{\star \star} P<0.01 ;{ }^{\star \star \star} P<0.001$. (b) 3D scaling plot from a Random Forest proximity matrix of the SNPs from 15 adhesins (using the first 3 principal components) for human (light blue), bovine (green), equine (dark blue) and chicken (red) isolates; the analysis identified host-specific DNA signatures by separating subpopulations of isolates from the same host.

valine contributed to the preferential binding of $S$. Typhi FimH to human cells, we mutagenized the representative fimH80 gene of $S$. Typhi by substituting its valine with an alanine. Bacteria expressing the mutated FimH80(V223A) allele demonstrated significantly decreased binding to all three human cells and increased binding to three of the four bovine cells investigated (Fig. 2c). Taken together, these results highlighted how unique nsSNPs in a bacterial adhesin from a broad-host range serovar contribute to bacterial-host interactions, and likely participate in host specificity.

fimH variants coincide with distinct host-adapted Salmonella. Based on our findings in the broad host-range serovar Typhimurium, we hypothesized that allelic variation of $f i m H$ would also associate with host specificity in more host-adapted serovars, potentially contributing to bacterial-host interactions that drive host adaptation. Although most S. enterica strains and serovars express type 1 fimbriae and some limited studies suggested fim $H$-host associations ${ }^{14,17,18}$, no systematic large-scale comparative study has yet linked fim $\mathrm{H}$ alleles to host-associated strains or serovars. Here, we collected 1,848 individual fimH genes from 76 different serovars (Supplementary Data 7), and identified a total of 152 unique fimH sequences for 105 different allelic FimH proteins with 166 amino-acid substitution sites and 1 three-nucleotide insertion. The phylogeny of fim $\mathrm{H}$ demonstrated a strong serovar-specific lineage, with strains of the same serovar clustering either together or in a few distinct branches, as illustrated for the major alleles (Fig. 3). Furthermore, a mutual information analysis confirmed a strong correlation between serovars, fimH alleles and FimH proteins, consistent with serovar lineage evolution in Salmonella ${ }^{19}$ (Supplementary Fig. 6). Positions of the major substituted amino acids were mapped on a proposed three-dimensional model of the Salmonella FimH that is based on the crystal structure of the FimH adhesin on the tip of the Escherichia coli type 1 fimbriae ${ }^{20,21}$. The model suggests an amino-terminal lectin domain that consists of 174 amino acids 

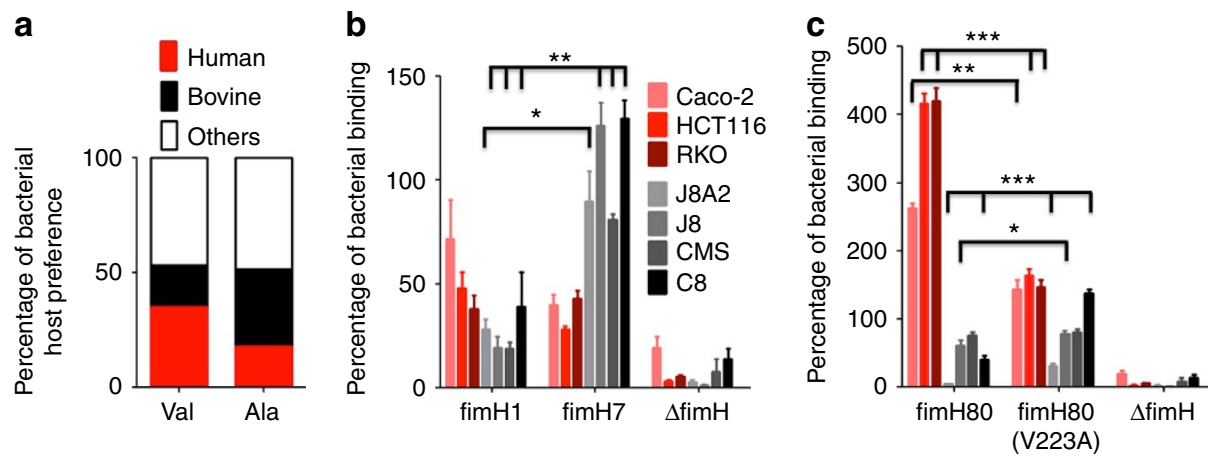

Figure 2 | Residue 223 variation in FimH of S. Typhimurium and Typhi swaps host specificity. (a) Host origin distribution for 580 S. Typhimurium isolates that have either a valine ( $\mathrm{Val}$ ) or an alanine (Ala) at position 223 of FimH. (b) Binding to three human (in red) and four bovine (in black) intestinal epithelial cells of recombinant E. coli expressing Salmonella type 1 fimbriae with the FimH1 or FimH7 alleles that have valine or alanine at position 223, respectively. (c) The different binding properties of fimH 80 with valine and engineered fimH 80 with alaline at position 223 for three human and four bovine enterocytes. The data in $\mathbf{b}$ and $\mathbf{c}$ are expressed as mean percentages of bacterial binding relative to the difference between fim 2 ( $100 \%$ binding for Fim $\mathrm{H}$ of $\mathrm{S}$. Typhimurium strain AJB3, not shown) and $\triangle \mathrm{fimH}$ ( $0 \%$ binding) with \pm s.e.m. of three experiments. P-values were calculated by using a one-sided paired $t$-test: ${ }^{\star} P<0.05 ;{ }^{\star \star} P<0.01 ;{ }^{\star \star \star} P<0.001$.
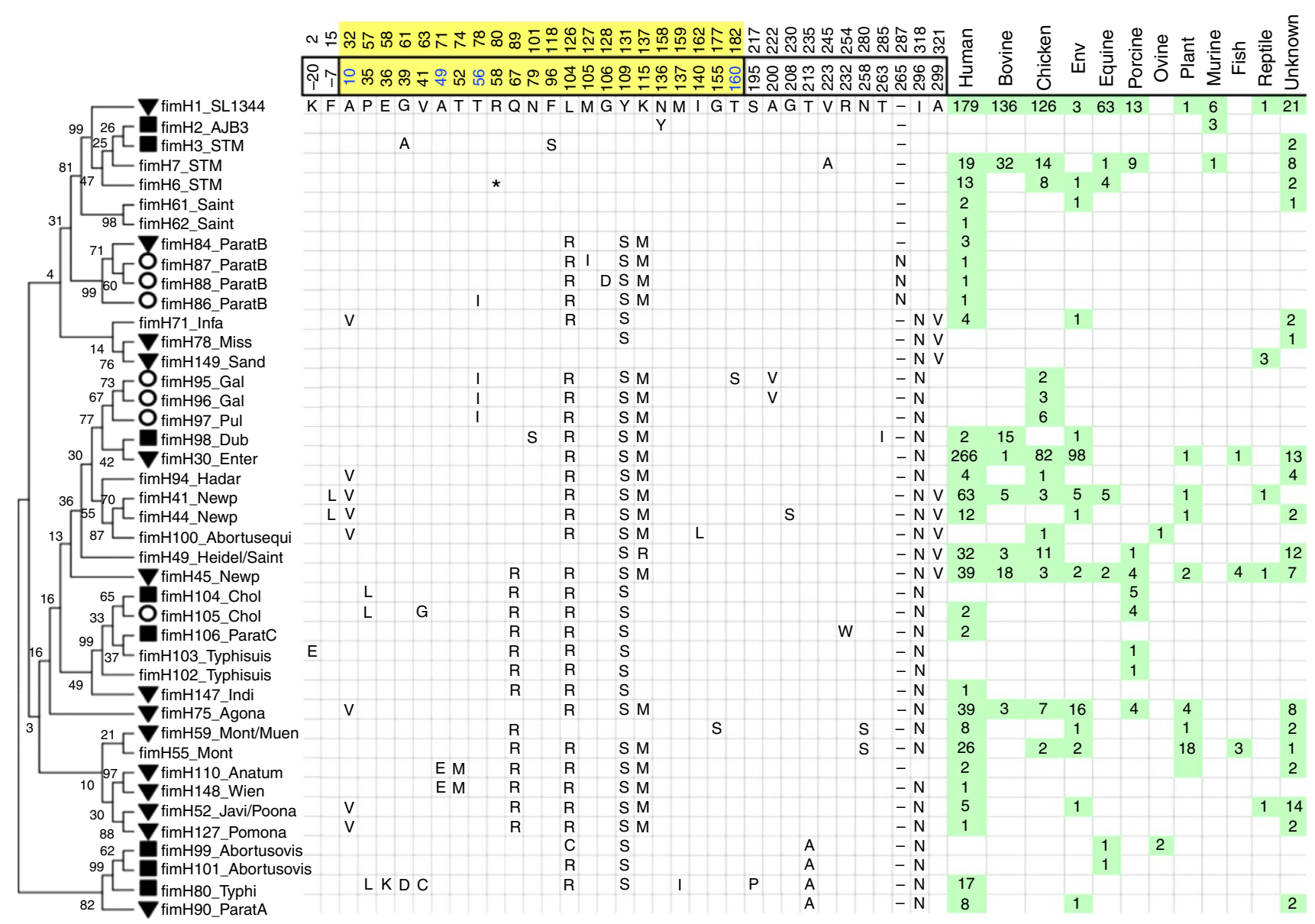

Figure 3 | FimH protein sequence variants. Variant residue positions for the unprocessed (top line) and matured (second line) FimH proteins, with the FimH of strain SL1344 (FimH1) used as the comparative standard (third line). The star for Typhimurium fimH6 is a stop codon. The signal peptide (22 residues), lectin (residues 1-173 of the mature protein, yellow background) and pilin domains (residues 177-315) are framed (second line). Substitutions and their corresponding positions are shown for each listed FimH. Variant residues that are predicted to participate in the mannose-binding pocket are highlighted in blue. At left, phylogenetic tree of the major fimH alleles (found in at least ten isolates per serovar; two, two and four isolates for serovars Typhisuis, Abortusovis and Abortusequi, respectively) based on nucleotide sequences and built by using the Maximum-likelihood method with a bootstrap value of 1,000. The mannose-binding properties of FimH are indicated as high binding (black square), low binding (black triangle) and nonbinding (white circle $)^{29}$. On the right highlighted in green are the numbers of isolates studied for each listed allele and their origin (host, environment or unknown). 


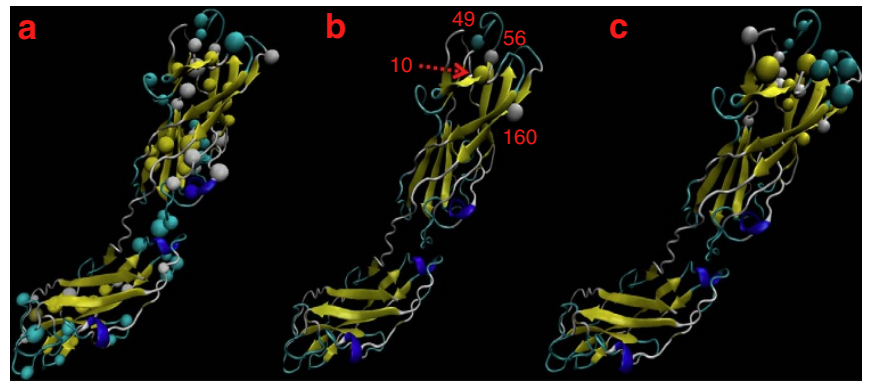

Figure 4 | Predicted structure of Salmonella FimH1 (S. Typhimurium SL1344). The balls highlight the substituted residues. (a) All the aminoacid residues found to be substituted, as listed in Fig. 3; (b) all the substituted residues in the predicted binding pocket (Fig. 3); (c) all the residues predicted to be involved in the mannose-binding pocket, including position $3,4,10,11,12,13,14,15,47,48,49,50,56,59,116,120,122,125$, $149,151,152,153,158,159,160$ and 162. $\alpha$-Helices were highlighted in dark blue, $\beta$-sheets in yellow, $\beta$-turns in grey and $\gamma$-turns in light blue in the predicted tertiary structure of FimH1.

and a carboxy-terminal pilin domain of 137-138 amino acids that anchors FimH at the fimbrial tip ${ }^{20-22}$. Among the major allelic FimH proteins, 4 substitutions were detected in the signal peptide, 43 in the predicted lectin domain and 28 in the pilin domain, as predicted from a FimH structural model (Fig. 4a). Interestingly, no mutations were found in the three residues predicted to form the short segment linking the amino-terminal lectin domain of FimH with its carboxy-terminal pilin domain ${ }^{21}$. The predominance of substitutions within the lectin domain in serovar Typhimurium strains (Supplementary Table 4) as well as all other serovar strains studied (Supplementary Table 5), suggested that these mutations were positively selected by recognition of new receptors or affinity maturation of existing receptors. Together, our results support a model of evolutionary adaptation of FimH ligands to host-specific receptors, potentially contributing to host-adaptation and pathogenesis.

Host-adapted Salmonella FimH bind in a host-specific manner. To evaluate the biological relevance of the in silico determined associations detected above, we assessed the host-specific binding properties of a collection of allelic FimH proteins from major serovars by expressing them in the context of Salmonella type 1 fimbriae on recombinant E. coli. Binding assays using human, bovine and porcine intestinal epithelial cells, and hepato-epithelial chicken cells, revealed that several allelic FimH proteins conferred significant host-specific binding (Supplementary Figs 7-10). The most impressive host-specific adhesion was exemplified by the fimH102 or fimH103 alleles from the porcinerestricted paratyphoid fever S. Typhisuis, and fimH104 of $S$. Choleraesuis, a porcine isolate of this typical porcine-adapted serovar. All three encoded allelic adhesins mediated significantly greater bacterial binding to the porcine enterocytes IPEC-J2 when compared with all the other allelic FimH proteins, including FimH2 of $S$. Typhimurium AJB3, which served as a baseline control (Fig. 5 and Supplementary Fig. 7). Consistent results were obtained with porcine enterocytes IPEC1, albeit the differences were less impressive. In contrast, all three allelic FimH from porcine isolates mediated poor bacterial binding to three human intestinal epithelial cells (Fig. 5 and Supplementary Fig. 8). However, $S$. Choleraesuis can also cause systemic infections in humans, and FimH105 from a human isolate of $S$. Choleraesuis mediated significantly better bacterial binding to the three human enterocytes than the allelic FimH proteins of the three porcine

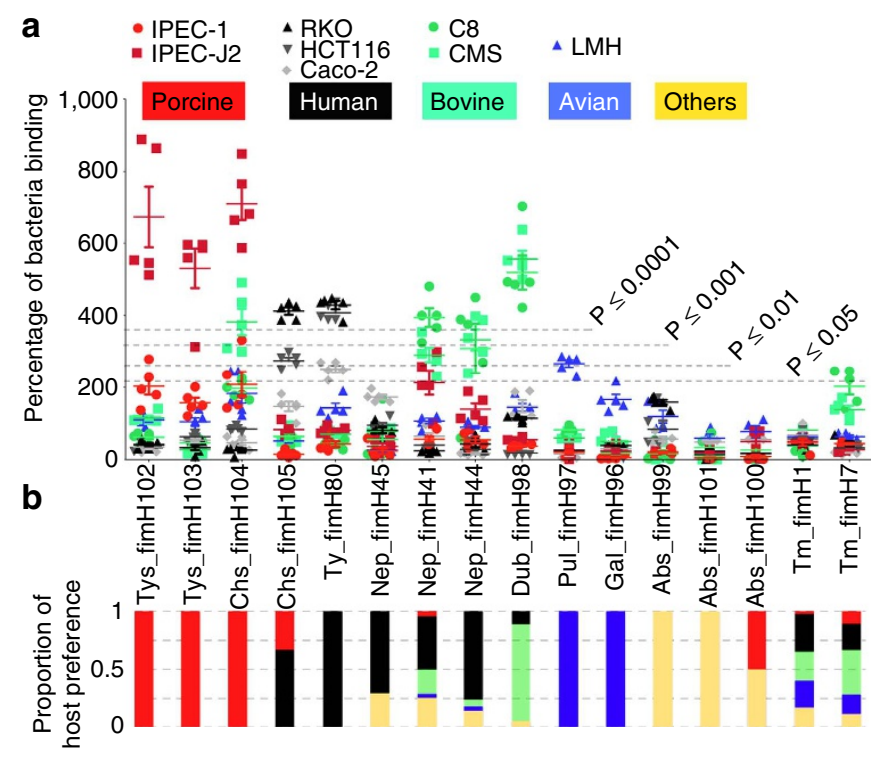

Figure 5 | FimH-mediated host-specific bacterial binding to enterocytes or hepato-epithelial cells. (a) Recombinant E. coli binding mediated by different Salmonella fimH-encoded allelic proteins with human (black), porcine (red) and bovine (green) enterocytes, as well as chicken hepatoepithelial cells (blue). The data are expressed as mean percentages of bacterial binding relative to the difference between fimH2 (100\% binding for FimH of S. Typhimurium strain AJB3, not shown) and $\triangle$ fimH ( $0 \%$ binding) with \pm s.e.m. of five experiments. $P$-values were calculated by the $t$-test that compared groups between each individual alleles and all the rest of data. The null hypothesis assumes a common binding affinity for all variants. The threshold of significance for the $P$-values indicated by grey dash-lines is as follows: 3.58 for $P \leq 0.0001 ; 3.16$ for $P \leq 0.001 ; 2.65$ for $P<0.01 ; 2.19$ for $P \leq 0.05$; and not shown, 1.94 for $P \leq 0.1$. (b) The bars for each fimH allele represent the proportion (1 representing 100\%) of Salmonella isolates from each corresponding host.

isolates. Conversely, fimbriated bacteria with FimH105 bound poorly to the two porcine intestinal epithelial cells (Supplementary Figs. 7). Notably, the distinct adhesive properties of $S$. Choleraesuis FimH104 and FimH105 were determined by a one amino-acid substitution (V41G), again highlighting the importance of nsSNPs in host specificity.

Both avian-restricted serovars Pullorum and Gallinarium FimH allelic proteins (FimH97 and FimH96, respectively) bound relatively better to chicken cells (Fig. 5 and Supplementary Fig. 9). Both FimH carry the T56I substitution that affects mannoseinhibitable binding 22 , but participates in the avian-specific binding property ${ }^{17}$. FimH98 of the bovine-adapted serovar mediated most efficient adhesion to the two bovine intestinal epithelial cells studied. Fimbriated bacteria with the allelic FimH99 and FimH101 proteins of S. Abortusovis, and FimH100 of $S$. Abortusequi did not bind to the human, bovine and porcine cells, possibly consistent with a preference for their respective hosts.

Although $S$. Newport has a broad host spectrum, it is frequently isolated from humans affected by foodborne infections, possibly because it is a major serovar isolated from cattle ${ }^{23}$. For this study, most of the S. Newport fimH sequences investigated were from human isolates, with the fimH41 and fimH44 being the most frequent, followed by a few fimH45 (Fig. 3). Only the former two alleles were present in bovine isolates. This association was consistent with the ability of bacteria expressing the corresponding proteins to bind best to bovine enterocytes (Fig. 5 and Supplementary Fig. 10) as 
compared with human enterocytes (Fig. 5 and Supplementary Fig. 8). In contrast, the fim 445 allele was absent from bovine isolates (Fig. 3) and fimbriated bacteria with FimH45 bound best to the human enterocytes, and particularly to Caco- 2 cells (Fig. 5 and Supplementary Figs 8 and 10).

Taken together, many allelic variants of FimH demonstrated distinct adherence preferences for host-specific enterocytes or hepatoepithelial cells, confirming the corresponding in silico detected associations between allelic adhesins and host specificity in a number of major Salmonella serovars. This extended study illustrates for the first time a molecular mechanism that likely contributes to host adaptation. Noticeably, the reported allelic variation of FimH causes a biologically relevant shift in adhesion that occurs both in broad-host range Salmonella, such as Typhimurium and Newport, and in host-adapted serovars in support of a significant functional role for nsSNPs in the evolutionary adaptation of the diverse Salmonella pathovars.

\section{Discussion}

Most studies of host adaptation by bacterial pathogens such as Salmonella focus either on a functional analysis of a specific gene $^{24}$ or utilize genomic comparisons to identify potential virulence genes ${ }^{4,25}$ but do not undertake subsequent functional assessments. The systematic approach used here determined that nsSNPs could potentially participate in the strain adaptation of Salmonella to individual host species. Our ability to identify specific sequence-determined host-adhesion properties that may contribute to pathodaptation to specific host-species resulted from a novel stepwise approach starting with a genomic comparison of 12 strains of serovar Typhimurium, the quintessential broad host range $S$. enterica serovar. Notably, SNPs in genes for membrane and surface-exposed proteins were among their most differentiating characteristics. In contrast, none of the other strain characteristics, such as the accessory and unique genes, pseudogenes, mobile DNA or genomic rearrangements bore any association with host specificity. A more stringent analysis of genes that encode $S$. Typhimurim surface proteins with known or suspected adhesive properties for colonization of host surfaces clearly highlighted associations between $S$. Typhimurium nsSNPs and specific hosts. This in silico result was further supported by the ability of FimH7, the most frequent FimH allelic adhesin of the Salmonella type 1 fimbriae from bovine isolates, to preferentially bind to bovine rather than human enterocytes (Fig. 2).

As an expansion of our investigation on FimH variants within another broad-range serovar, FimH of $S$. Newport, further illustrated nsSNPs effects on host-preferences. The bovine isolates of serovar Newport had two fim $\mathrm{H}$ alleles for proteins that bound best to bovine cells, whereas the third fimH was mainly present in human isolates and encoded a FimH adhesin that bound best to human enterocytes (Fig. 5 and Supplementary Figs 8 and 10). In addition, the most impressive host-specific interactions were observed with the FimH adhesins of host-adapted systemic serovars. For example, a detailed analysis of allelic variants of FimH in the swine-adapted serovar Choleraesuis, which can cause systemic diseases in humans, identified allelic adhesins that preferentially bind to intestinal epithelial cells of either humans or swine (Fig. 5 and Supplementary Figs 7 and 10). The host-specific adhesive properties of the FimH allelic proteins corresponded significantly with the host origin of the Salmonella strains that carried the respective fimH allele, verifying their physiologic relevance in vivo. Noticeably, FimH of known host-associated serovars, such as Typhi, Dublin, Gallinarum, Pullorum and Cholereasuis, had at least one fimH allele for an adhesin that mediated preferential bacterial binding to the epithelial cells of their respective hosts (Fig. 5 and Supplementary Figs 7-10). Thus, functional analysis of 21 allelic FimH adhesins confirmed the participation of these proteins in host-specific binding, strongly suggesting that preferential adhesion to relevant mammalian or avian cells participates in the evolutionary adaptation to specific hosts, even within individual serovars. These relevant associations were detected despite potential confounding effects, such as the likely inclusion of Salmonella strains from broad host serovars transiently passaging in non-preferential hosts, the polygenic nature of host-adaptive evolution, or nsSNPs responsible for neutral substitutions, highlighting the power of the approach.

Our finding that all mammal-specific FimH allelic proteins identified bound intestinal cells of their hosts in a mannoseinhibitable manner was somewhat surprising, as it is currently unclear how binding of mannose residues on glycoprotein receptors could contribute to host specificity. We predict that the differential ability of FimH adherence to bind to various host cells is due to the density, accessibility, flexibility, orientation or length of the diverse mannose-bearing oligosaccharides on host cell-specific receptors ${ }^{18,26}$. Indeed, such factors may explain why simple in vitro binding assays that classify FimH adhesins according to their binding affinity for mono-, tri- or pentamannose model receptors, or adhesion to yeast or non-intestinal human cell lines ${ }^{27-31}$ do not always reflect their host-specific binding to intestinal cells. For example, we found that the serovar Typhisuis FimH, which is identical to the serovar Indiana FimH reported to have low-affinity binding in vitro ${ }^{29}$, bound strongly to the porcine enterocytes (Fig. 5 and Supplementary Fig. 7). Similarly, Choleraesuis FimH105 (V41G) and Newport FimH45, which do not bind mannose in vitro ${ }^{29}$, bound well to human intestinal cells, with the latter binding best to human Caco 2 cells (Fig. 5 and Supplementary Fig. 8). Thus, our results support the critical importance of utilizing physiologically relevant cells to functionally characterize pathogen-host interactions.

Although the binding of all mammalian FimH allelic proteins is mannose-sensitive, the specific binding of FimH adhesins from avian Pullorum and Gallinarum to chicken leukocytes is not mannose-inhibitable, indicating that this FimH allele recognizes a distinct receptor ${ }^{17}$. Here, we confirmed the avian-specificity and relative insensitivity to mannose inhibition of the Gallinarum and Pullorum FimH, with the incomplete inhibition observed likely reflecting the use of alpha-methyl-D-mannoside, a modified and powerful inhibitor, in excess (Fig. 5 and Supplementary Fig. 9). Notably, the FimH amino-acid substitution T56I in Gallinarum, Pullorum and chicken isolates of Paratyphi B with the fimH56 allele (Figs 3,4b and 6f) is a strong determinant for avian specificity $17,22,29,32$. Mutation of the avian fimH to substitute an isoleucine with a threonine at position 56 , restores its ability to bind a mannosylated glycoprotein ${ }^{22}$, and a Gallinarum strain engineered to express such a mannose-binding FimH was significantly less invasive in chicks ${ }^{32}$, supporting the idea that this single amino-acid substitution determines both mannosebinding and avian host specificity. In addition, Gallinarum and Pullorum adhered to and invaded mammalian cells only when engineered to express the Typhimurium type 1 fimbriae with a threonine in position 56 of FimH $^{33}$. Taken together, our results corroborate the importance of the FimH amino-acid substitution at position 56 to make a jump between mammalian and avian hosts. Further, our data confirm that a single amino-acid substitution in the binding pocket of FimH permits a switch in host specificity based on the presentation of mannose or nonmannose receptors by mammalian or avian hosts, respectively.

Although nsSNPs were found throughout mammalian-adapted FimH sequences, they were more abundant in the lectin domain, many surrounding the binding pocket (Fig. 4c), suggesting a direct role in binding. A comparison of these sequences identifies 


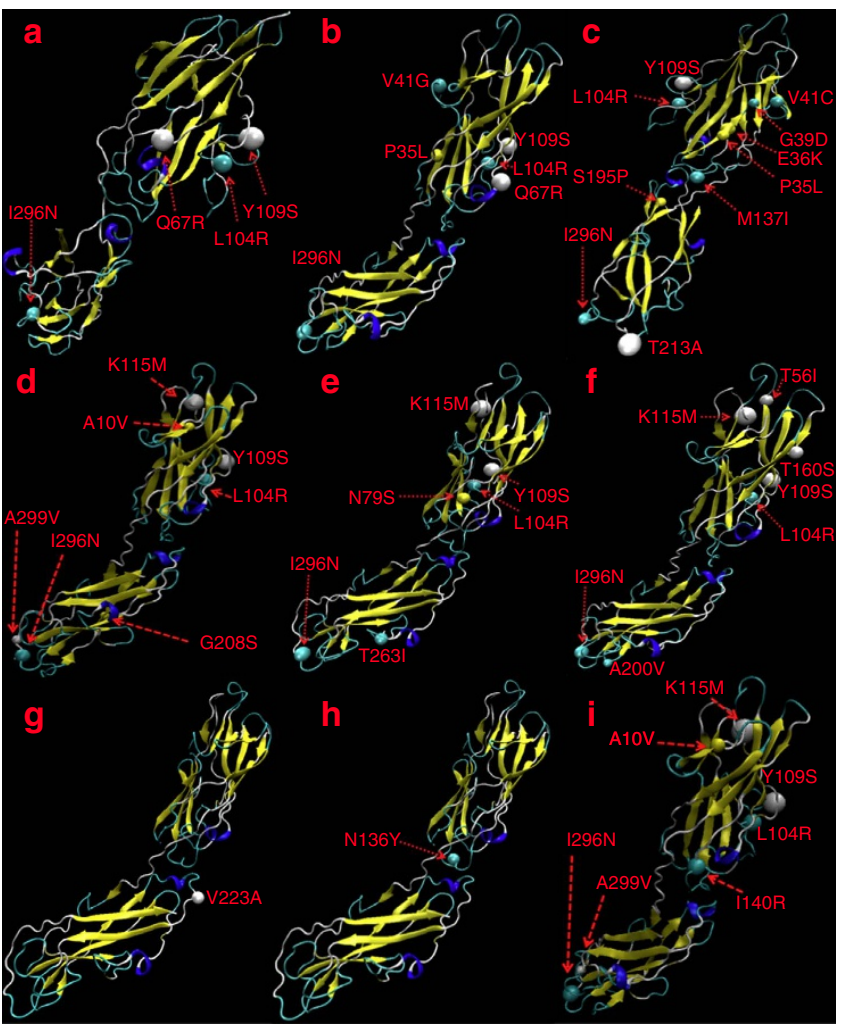

Figure 6 | Substituted sites on FimH alleles visualized on the Salmonella FimH model (S. Typhimurium SL1344). The balls highlight the substituted residues. (a) Serovar Typhisuis FimH102 or FimH103; (b) serovar Choleraesuis FimH105; (c) serovar Typhi FimH80; (d) serovar Newport FimH44; (e) serovar Dublin FimH98; (f) serovar Gallinarum FimH95; (g) serovar Typhimurium FimH7; (h) serovar Typhimurium FimH2;

(i) serovar Abortusequi FimH100. $\alpha$-Helices were highlighted in dark blue, the $\beta$-sheets in yellow, the $\beta$-turns in grey and the $\gamma$-turns in light blue in the predicted tertiary structure of FimH1.

amino acids that most likely contribute to host-specific binding. For example, substitutions in the lectin domain, such as E36K and V41C of the Typhi FimH80 likely alter the conformation of the binding pocket to promote human specificity (Figs 3 and $6 c$ ). FimH80 also shares a substituted V41 with Choleraesuis FimH105 (albeit V41C versus V41G) from a human isolate, suggesting that position 41 of FimH has a role in human specificity (Fig 6a-c). Similarly, the Q67R substitution likely promotes host adaptation of porcine-associated serovars such as Typhisuis and Choleraesuis, but not of bovine-associated serovars such as Dublin (Fig. 6e) and Newport (Fig. 6d), with the exception of the human-adapted FimH45 of Newport (Fig. 3). Thus, a selective group of amino-acid residues within the FimH lectin domain appears to play a dominant role in determining host-specific binding by the adhesin. Moreover, residues in these positions must influence FimH binding indirectly, as they are not found within the binding pocket.

Most surprising were variable FimH amino-acid residues that resided outside of the lectin domain but still affected host-specific adhesion. For example, several variable residues around the linker domain increased binding to mannose, as confirmed with the natural FimH2 N136Y substitution (Figs 3 and $6 \mathrm{~h})^{17,34}$. Linker domain substitutions in natural FimH also modulated host specificities, as shown with the V223A substitution of FimH7, which increased the binding affinity for bovine cells (Fig. 6g). Similarly, the I140L substitution in FimH100 of Abortusequi abrogated the binding to bovine cells detected with Newport (Fig. 6d,i). To a certain extent, these findings are consistent with a previous random mutagenesis study that revealed the role of the linker domain in allosteric effects on FimH-mediated adhesion $^{34,35}$. When FimH is subjected to tensile force, it undergoes structural changes in which an extended linker domain allows the pilin and lectin domains to separate, forming a binding pocket that closes around the mannose receptor like a Chinese finger trap ${ }^{20}$. Thus, one can speculate that residue substitutions in the linker domain could indirectly lead to conformational changes in the binding pocket to influence FimH host-specific binding. Finally, it is possible that stepwise mutations, some near the linker domain, participate in the evolution of host adaptation with the addition of individually silent mutations affecting adhesion in a cumulative or epistatic manner.

Collectively, our results on the function of FimH variants illustrate the significance of nsSNPs as a molecular mechanism by which Salmonella expands its host range. Most significantly, this study provides the first use of a genome-wide association study to prompt a subsequent systematic functional analysis, which revealed an evolutionary positive selection process that may contribute to host adaptation of Salmonella. Although our results need in vivo confirmation using corresponding animal models, our current in vitro identification of host-specific allelic variants in surface molecules should provide the basis for future diagnostic assays of host-specific pathogens and may allow for the development of anti-adhesive antimicrobials that interfere with host-specific intestinal colonization and invasion. Moreover, this study opens the possibility that allelic variation in a wide range of bacterial proteins that participate directly in virulence, or indirectly by modulating metabolic or regulatory pathways ${ }^{10}$ may also contribute to host specificity and pathogenesis. As such, this work has broad implication in the field of bacterial pathogenesis, as the used approach can identify and assess the role of specific allelic variants in any pathogen for which groups of isolates with relevant metadata and appropriate functional tests exist.

\section{Methods}

Bacterial strain and data collection. A total of 382 Salmonella Typhimurium strains were isolated in the United States America between 1988 and 2010 from different hosts or from the environment. Human and food isolates were obtained from collections at the US Centers of Disease Control and Prevention (CDC) and the US Food and Drug Administration (FDA), respectively, whereas animal isolates were from the Salmonella Reference Center at the University of Pennsylvania (UPENN). All the Salmonella Typhimurium isolates were identified by standard serotyping methods, using $\mathrm{O}$ - and $\mathrm{H}$-antigen agglutination, based on the Kauffmann-White Scheme ${ }^{36}$. Isolates were grown on LB (Lennox) medium, and single colonies were incubated in LB broth overnight at $37^{\circ} \mathrm{C}$. Bacterial cells were pelleted by centrifugation $(3,700 \mathrm{~g}$ for $10 \mathrm{~min})$ and DNA was extracted using the Wizard SV 96 Genomic DNA Kit (Promega) according to the manufacturer's instructions. DNA quality and quantity were evaluated by gel electrophoresis and determined with a NanoDrop 1,000 spectrophotometer (Thermo Fisher Scientific).

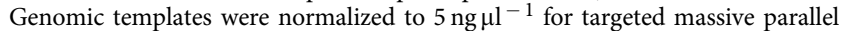
sequencing, as described below. An additional 12 complete genomes with metadata of serotype Typhimurium were downloaded from GenBank and sequence data from 186 Typhimurium genomes were collected from the NCBI SRA database. All 580 Typhimurium isolates are listed in Supplementary Tables 3 and 4 . A total of 1,268 additional individual fim $H$ gene sequences from 76 serovars with metadata of the corresponding strains were extracted from six publications ${ }^{29,37-41}$ as well as new sequences produced from our lab.

DNA sequencing. For targeted massive parallel sequencing, primer pairs for 15 genes, including the genes for 12 fimbrial adhesins (StcD, SafD, BcfD, FimH, StbD, SthE, StdD, StiH, StfH, LpfD, StjA, PefA $)^{21}$ and 3 outer-membrane proteins (OmpA, OmpC, OmpN) were designed and synthesized (Integrated DNA

Technologies, Inc.) with 3-4 primer pairs per gene (Supplementary Table 6). The sequencing libraries were prepared using the Access Array system (Fluidigm South San Francisco $)^{14}$. Quality and quantity of the amplicon libraries were evaluated with a 2,100 Bioanalyzer instrument (Agilent Technologies) and NanoDrop. The libraries were pooled in equal amounts for pyrosequencing with a 454 GS FLX 
sequencer using Titanium chemistry (454 Life Sciences, Roche) at the DNA Sequencing Facility of UPENN. An in-house Perl script was used for sequence splitting and barcode removal. Sequence assembly and mapping were done with SeqMan (DNASTAR, Inc.). A total of 15 genes of 382 strains were sequenced with a coverage of more than 30 and a Phred quality score of more than 40 for data analysis. For Sanger DNA sequencing, the fimH gene of 210 clinical isolates from various $S$. enterica serovars were amplified with the Pfu polymerase (New England Biolabs Inc.) and each individual gene sequence was assembled using at least three sequencing reads to get a Phred quality score of more than 30 .

Genomic analysis. Mauve ${ }^{42}$ was used for comparative analysis of 12 sequenced Typhimurium full genomes. A core genome was assigned by using thresholds of $95 \%$ sequence identity and 95\% sequence length coverage. Comparative circle map and gene functional categories were determined by the rapid annotation of microbial genomes using subsystems technology ${ }^{43} . P$-value associated with a functional category measured the likelihood that the association between a set of genes with SNPs and a given functional category is due to random chance. The smaller the $P$-value, the less likely the association was random and the more significant the association. In general, $P$-values less than 0.05 indicated a statistically significant, non-random association. $P$-values were calculated using the right-tailed Fisher exact test (Prism, GraphPad Software, Inc.). All proteins with less than 50 amino-acid residues were removed from the core genome and pangenome analysis. The phage genes from the Typhimurium genome were determined by PHAST $^{44}$. All the pseudogenes were assigned by original genome annotation of the 12 complete Typhimurium genomes under manual correction. A total of 186 strains of Typhimurium with SRA data produced by Illumina pairedend technology were submitted for de novo genome assembly and annotation by using CloVR ${ }^{45}$. CloVR used both virtual machine and cloud computation technology for high-throughput data processing. All the annotated genomic data were uploaded into $\mathrm{SEED}^{43}$ for further data storage, sequence extraction and analysis. The 15 genes studied for all the 186 strains had quality score of more than 30. SNPfinder ${ }^{46}$ was used to detect the SNPs for the $12 \mathrm{~S}$. Typhimurium complete genomes.

Population and phylogenetic analysis. DnaSP5 (ref. 47) was used to estimate several measures of DNA sequence variation within and between populations, including neutrality analysis (Tajima's D) and SNP detection. Nucleotide diversity estimates $^{48}$ and recombination analyses were carried out by using DataMonkey ${ }^{49}$. The homoplasious SNPs, that is, SNPs due to recombination and horizontal gene transfer, were detected by using START2 (ref. 50) with a threshold of 0.6 .

\section{Statistics and association analysis. All the SNPs and their corresponding} metadata were used for association studies. The Akaike Information Criterion (AIC) of the multinomial logit (logistic regression) model was used for testing the genetic association of multiple phenotypes with the 'nnet' $\mathrm{R}$ package ${ }^{15}$ and the following equation: $\mathrm{AIC}=-2 \log L+2((k-1)+s)$, where $k$ is the number of levels of the dependent variable and $s$ is the number of predictors in the model. The model with the smallest AIC was considered the best. The resulting association coefficient $e$ was determined as followed: $\mathbf{e}=(y f t)(y t f)^{\wedge}-1(y=$ number of correct predictions, $y t=$ total number of correction prediction, $f=$ number of false prediction, $f t=$ total number of false prediction). MultiPhen ${ }^{16}$ identified the linear combination of traits most associated with each genetic variant by applying a reversed ordinal regression, such that genotype (allele count) is regressed on a collection of traits. The test for association was a likelihood ratio test for model fit, testing whether all regression coefficients in the model were jointly significantly different from zero. RF was used to identify key SNPs involved in the studied associations (SPM v7.0, Salford Systems). The three-dimensional scatterplot (Fig. 1b and Supplementary Fig. 4) of the multi-dimensional scaling coordinates were obtained from the RFs proximity matrix. All heat-map images were produced by using the neighbour-joining method for hierarchical clustering of $\mathrm{MeV}$ with 1,000 bootstrap replicates ${ }^{21}$. Multivariate mutual information statistics was used to detect and evaluate the dependency among $f i m H$ alleles, FimH alleles, serovars and host origins ${ }^{51}$. The maximum likelihood estimators of Shannon's entropy and multiple mutual information were obtained by sampling the system variables and using the resulting empirical values of probability distributions.

Structure-function analysis. Processed FimH allele 1 (FimH1) from strain SL1344, which comprises 291 amino acids (or 313 residues minus its 22 residue long signal peptide), was used for modelling the structure. The secondary structure of FimH was predicted by machine learning with the I-TASSER server ${ }^{52}$. The best template structure was identified by matching the protein sequence and predicted secondary structure with the solved structure (1klf, PDB library), using LOMET threading ${ }^{53}$. The mannose-binding site was mapped by matching the predicted structure with structures of the PDB function library using $\mathrm{COACH}^{54}$. The structure was edited and visualized with $\mathrm{VMD}^{55}$. The functional data for binding properties to mannose receptors were from the following papers ${ }^{17,18,29}$.
Site-directed mutagenesis and recombinant engineering. The construction of recombinant $E$. coli that express different Salmonella fimH alleles was done as follows. Different fimH alleles were amplified using the high-fidelity Pfu DNA polymerase with appropriate primers (Supplementary Table 14) and templates from the desired strains, restricted with $N d e \mathrm{I}$ and $\mathrm{BamHI}$, and ligated to the correspondingly restricted pMAL-c2X plasmid (New England Biolabs, Inc.). All the fim $H$ were sequenced by Sanger Sequencing to confirm correct amplification (UPENN, DNA Sequencing Facility). E. coli AAEC189, which lacks the E. coli fim gene cluster ${ }^{56}$ and carries plasmid pAZ37 that contains the S. Typhimurium fim gene cluster with no functional fim $\mathrm{H}^{17}$, was used to transform with plasmids expressing the different Salmonella FimH alleles. Expression of the type 1 fimbriae and mannose-specific recognition by FimH were determined by slide agglutination with anti-type 1 fimbriae antisera (seroagglutination) and by yeast cell

(Saccharomyces cerevisiae) aggregation, respectively. The site-directed mutant of fimH, fimH80 was used as template for overlap PCR with primer pairs (forward: 5'-AAATGTACCAACGCCGCGGCGCAGGCCTATTTATC-3'; reverse: $5^{\prime}$-GATAAATAGGCCTGCGCCGCGGCGTTGGTACATTT- $3^{\prime}$ ) targeting the corresponding position. The mutated fim $\mathrm{H}$ amplicon was cloned into pMAL-c2X, sequenced to confirm correct amplification and introduced into AAEC189.

Eukaryotic cell cultures. The human colonic cell lines Caco-2 (ATCC HTB-37) HCT116 (ATCC CCL247) and RKO (ATCC CRL2577) were obtained from the NIH/NIDDK Digestive Diseases Center at UPENN. All three cell lines were routinely cultured in Dulbecco's Modified Eagle Medium (DMEM; Invitrogen, Life Technologies) supplemented with $20 \%(\mathrm{v} / \mathrm{v})$ fetal bovine serum (FBS), $1 \%(\mathrm{v} / \mathrm{v})$ non-essential amino acids and antibiotics to a final concentration of $100 \mathrm{Uml}^{-1}$ penicillin and $100 \mu \mathrm{g} \mathrm{ml}^{-1}$ streptomycin (Gibco, Life Technologies). The porcine

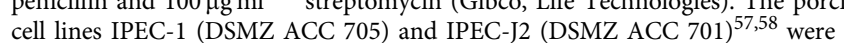
cultured with 10\% FBS (Sigma-Aldrich), 1\% penicillin/streptomycin, 1\% insulin/ transferrin/selenium (Gibco), and $5 \mathrm{ng} \mathrm{ml}^{-1}$ epidermal growth factor (Sigma) in DMEM/F-12/HAM (1/1/1, v/v/v; Gibco). The bovine intestinal epithelial cells were immortalized with the thermo-sensitive mutant of a retrovirus vector coding for the SV40 large T-antigen oncogene, using standard procedures, after isolation by enzymatic (C8, J8 and J8A2 cell lines) or Matrisperse dissociation (CMS cell line) of the intestinal mucosal segments ${ }^{59-61}$ (personal communication from Jacques Mainil). The cells were seeded on the surfaces of plates pretreated with bovine collagen (95\% type I, Vitrogen 0701/FXP-019, $1 \mu \mathrm{g} \mathrm{cm}^{-2}$; Nutacon BV), using OptiMEM medium (Gibco) with $1 \%(\mathrm{v} / \mathrm{v})$ of a mixture of antibiotics and antimycotic (Gibco), $0.2 \%$ of bovine pituitary gland extract (Gibco), $1 \%$ of insulin-transferrin-selenium (Gibco), 1\% of glutamax (Gibco), 1\% of sodium pyruvate (Sigma), $10 \mathrm{nM}$ hydrocortisone (Sigma), $20 \mathrm{nM}$ triiodothyronine (Sigma), $10 \mathrm{ng} \mathrm{ml}^{-1}$ epidermal growth factor (Sigma), $10 \mu \mathrm{g} \mathrm{ml}^{-1}$ linoleic acid-albumin from bovine serum albumin (Sigma), $1 \%(\mathrm{v} / \mathrm{v})$ nonessential amino acids (Gibco) and $1 \%(\mathrm{v} / \mathrm{v})$ FBS (Hyclone). The avian cell line LMH (ATCC CRL-2117 ${ }^{\mathrm{TM}}$ ) was seeded on $0.1 \%$ gelatin-coated plate and cultured in Waymouth's MB 752/1 medium (Gibco) with $10 \%(\mathrm{v} / \mathrm{v})$ FBS and antibiotics $\left(100 \mathrm{U} \mathrm{ml}^{-1}\right.$ penicillin and $100 \mu \mathrm{g} \mathrm{ml}^{-1}$ streptomycin, Gibco). All the cells were incubated at $37^{\circ} \mathrm{C}$ in a humid atmosphere containing $5 \%$ of $\mathrm{CO}_{2}$ or $10 \% \mathrm{CO}_{2}$ for the bovine cells.

Bacterial binding assays. Human, porcine, bovine and chicken epithelial cell cultures were used for the binding assays with recombinant E. coli AAEC189 $\left(\Delta\right.$ fim $\left._{\text {E.coli }}\right)$ expressing Salmonella type 1 fimbriae with different FimH alleles. The bacteria were grown under static condition for $48 \mathrm{~h}$. All the bacteria were washed three times with PBS, and diluted in DMEM to $10^{7} \mathrm{CFU} \mathrm{ml}{ }^{-1}$ before use. The overnight seeded eukaryotic cells were grown to monolayers in 96-well plates (Corning, CLS3596). Bacteria were added at a multiplicity of infection of 200 to and allowed to interact with the cells for $1 \mathrm{~h}$ at $37^{\circ} \mathrm{C}$ in $5 \% \mathrm{CO}_{2}$. The cells were then washed five times with PBS and lysed with $1 \%$ Triton (Sigma). The number of $\mathrm{CFU}$ in each well was quantified by plating serial dilutions of cell lysates on LB plates. For inhibition, bacterial binding was tested in the presence of $50 \mathrm{mM}$ methyl-D-mannopyranoside $(\alpha-\mathrm{mm})$. Binding data were the results of five individual repeated experiments. Bacterial binding was compared with the binding of recombinant E. coli that expressed the FimH3 (FimH of strain AJB3) set at 100\% and the binding of recombinant bacteria that expressed no fim $\mathrm{H}$, set at $0 \%$. Percentage of bacterial binding mediated by FimH allele X (X representing any specific FimH allele studied) was calculated in the following way: \% bacterial binding with $\mathbf{X}=\left(\mathrm{CFU}_{\mathrm{X}}-\mathrm{CFU}_{\mathrm{AJB} 3}\right)\left(\mathrm{CFU}_{\mathrm{AJB} 3}-\mathrm{CFU}_{\Delta \mathrm{fimH}}\right)^{\wedge}-1$. The $P$-value was calculated by the $t$-test that compared groups between each individual alleles and all the rest of data. The null hypothesis assumed a common binding affinity for all variants.

Analysis of fimbriae expression. Recombinant fimbrial expression on E. coli was determined by two methods. First, a semi-quantitative standard slide seroagglutation test with anti-fimbrial antisera was used to check for fimbriation ${ }^{17}$ Second, the relative amount of fimbriae on bacteria was measured quantitatively by two parallel twofold serial dilution ELISAs, one assay measuring the level of bacterial fimbriation, and the other controlling the level of coated bacteria in the plastic wells. For this, fimbriated E. coli AAEC189 expressing Salmonella type 1 fimbriae were washed three times in PBS, adjusted to an OD600 of 1.0 and coated on 96 -well microlitre plate, followed by blocking with $1.5 \%$ BSA. Bacterial coating 
efficiency was quantitated with biotinylated anti-E. coli antibodies $\left(10^{-2}\right.$, Pierce PA1-73035, Thermo Fisher Scientific) and the amount of expressed fimbriae, with anti-fimbriae antisera $\left(5 \times 10^{-2}\right)$ followed by Streptavidin HRP conjugate (Pierce) or ECL anti-rabbit IgG HRP $\left(2 \times 10^{-3}\right.$, GE Healthcare Bio-Sciences, RPN4301), and 1-Step Turbo TMB-ELISA Substrate Solution (Pierce), with standard PBS washes in-between each step. Reactions were stopped with $2 \mathrm{M} \mathrm{H}_{2} \mathrm{SO}_{4}$ and absorbance $(A=450 \mathrm{~nm})$ was determined with a Synergy HT Multi-Mode Microplate Reader (BioTek). Anti-bacteria and -fimbriae antibody dilutions giving $50 \%$ binding $\left(A_{50}\right)$ for each fimbriated bacteria were used to calculate relative amounts of fimbriae per bacteria $\left(\right.$ fimbria $\left._{\mathrm{x}}\left(A_{50}\right)\right)\left(\operatorname{bacteria}_{\mathrm{x}}\left(A_{50}\right)\right)^{\wedge}-1$. The experiments were repeated three times.

\section{References}

1. Shi, Y., Wu, Y., Zhang, W., Qi, J. \& Gao, G. F. Enabling the 'host jump' structural determinants of receptor-binding specificity in influenza A viruses. Nat. Rev. Microbiol. 12, 822-831 (2014).

2. de Graaf, M. \& Fouchier, R. A. Role of receptor binding specificity in influenza A virus transmission and pathogenesis. EMBO J. 33, 823-841 (2014).

3. Spano, S. \& Galan, J. E. A Rab32-dependent pathway contributes to Salmonella typhi host restriction. Science 338, 960-963 (2012).

4. Chaudhuri, R. R. et al. Comprehensive assignment of roles for Salmonella typhimurium genes in intestinal colonization of food-producing animals. PLoS Genet. 9, e1003456 (2013).

5. Deng, L. et al. Host adaptation of a bacterial toxin from the human pathogen Salmonella Typhi. Cell 159, 1290-1299 (2014).

6. Mather, A. E. et al. Distinguishable epidemics of multidrug-resistant Salmonella Typhimurium DT104 in different hosts. Science 341, 1514-1517 (2013).

7. McClelland, M. et al. Comparison of genome degradation in Paratyphi A and Typhi, human-restricted serovars of Salmonella enterica that cause typhoid. Nat. Genet. 36, 1268-1274 (2004).

8. Langridge, G. C. et al. Patterns of genome evolution that have accompanied host adaptation in Salmonella. Proc. Natl Acad. Sci. USA 112, 863-868 (2015).

9. Viana, D. et al. A single natural nucleotide mutation alters bacterial pathogen host tropism. Nat. Genet. 47, 361-366 (2015).

10. Yue, M. \& Schifferli, D. M. Allelic variation in Salmonella: an underappreciated driver of adaptation and virulence. Front. Microbiol. 4, 419 (2014).

11. Kingsley, R. A. et al. Genome and transcriptome adaptation accompanying emergence of the definitive type 2 host-restricted Salmonella enterica serovar Typhimurium pathovar. MBio 4, e00565-13 (2013).

12. Okoro, C. K. et al. Intracontinental spread of human invasive Salmonella Typhimurium pathovariants in sub-Saharan Africa. Nat. Genet. 44, 1215-1221 (2012).

13. Parsons, B. N. et al. Invasive non-typhoidal Salmonella typhimurium ST313 are not host-restricted and have an invasive phenotype in experimentally infected chickens. PLoS Negl. Trop. Dis. 7, e2487 (2013).

14. Yue, M. et al. Combined with pyrosequencing for identification of allelic variants with phenotypic associations among targeted Salmonella Genes. Appl. Environ. Microbiol. 78, 7480-7482 (2012).

15. Venables, W. N. \& Ripley, B. D. Exploratory Multivariate Analysis in Modern Applied Statistics with S, 4th edn, 301-330 (Springer, 2002).

16. O'Reilly, P. F. et al. MultiPhen: joint model of multiple phenotypes can increase discovery in GWAS. PLoS ONE 7, e34861 (2012).

17. Guo, A. et al. FimH alleles direct preferential binding of Salmonella to distinct mammalian cells or to avian cells. Microbiology 155, 1623-1633 (2009).

18. Grzymajlo, K. et al. FimH adhesinrom host unrestricted Salmonella Enteritidis binds to different glycoprotein ligands expressed by enterocytes from sheep, pig and cattle than FimH adhesins from host restricted Salmonella Abortus-ovis, Salmonella Choleraesuis and Salmonella Dublin. Vet. Microbiol. 166, 550-557 (2013).

19. Soyer, Y., Orsi, R. H., Rodriguez-Rivera, L. D., Sun, Q. \& Wiedmann, M. Genome wide evolutionary analyses reveal serotype specific patterns of positive selection in selected Salmonella serotypes. BMC Evol. Biol. 9, 264 (2009).

20. Kisiela, D. I. et al. Allosteric catch bond properties of the FimH adhesin from Salmonella enterica serovar Typhimurium. J. Biol. Chem. 286, 38136-38147 (2011).

21. Yue, M. et al. Diversification of the Salmonella fimbriae: a model of macro- and microevolution. PLoS ONE 7, e38596 (2012).

22. Kisiela, D. et al. Characterization of FimH adhesins expressed by Salmonella enterica serovar Gallinarum biovars Gallinarum and Pullorum: reconstitution of mannose-binding properties by single amino acid substitution. Infect. Immun. 73, 6187-6190 (2005)

23. Waltman, D. \& Sellers, R. in Report of the Committee on Salmonella, Proceedings 117th Annual Meeting of the United States Animal Health Association, 328-338 (USAHA, Saint Joseph, MO, 2013).

24. Tsolis, R. M. et al. Identification of a putative Salmonella enterica serotype typhimurium host range factor with homology to IpaH and YopM by signature-tagged mutagenesis. Infect. Immun. 67, 6385-6393 (1999).
25. Barquist, L. et al. A comparison of dense transposon insertion libraries in the Salmonella serovars Typhi and Typhimurium. Nucleic Acids Res. 41, 4549-4564 (2013).

26. Karlsson, K.-A., Ångström, J., Bergström, J. \& Lanne, B. Microbial interaction with animal cell surface carbohydrates. APMIS 100, 71-83 (1992).

27. Boddicker, J. D., Ledeboer, N. A., Jagnow, J., Jones, B. D. \& Clegg, S. Differential binding to and biofilm formation on, HEp-2 cells by Salmonella enterica serovar Typhimurium is dependent upon allelic variation in the fimH gene of the fim gene cluster. Mol. Microbiol. 45, 1255-1265 (2002).

28. Dwyer, B. E., Newton, K. L., Kisiela, D., Sokurenko, E. V. \& Clegg, S. Single nucleotide polypmorphisms of fimH associated with adherence and biofilm formation by serovars of Salmonella enterica. Microbiology 157, 3162-3171 (2011).

29. Kisiela, D. I. et al. Evolution of Salmonella enterica virulence via point mutations in the fimbrial adhesin. PLoS Pathog. 8, el002733 (2012).

30. Misselwitz, B. et al. Salmonella enterica serovar Typhimurium binds to HeLa cells via Fim-mediated reversible adhesion and irreversible type three secretion system 1-mediated docking. Infect. Immun. 79, 330-341 (2011).

31. Grzymajlo, K., Kuzminska-Bajor, M., Jaworski, J., Dobryszycki, P. \& Ugorski, M. The high-adhesive properties of the FimH adhesin of Salmonella enterica serovar Enteritidis are determined by a single F118S substitution. Microbiology 156, 1738-1748 (2010).

32. Kuzminska-Bajor, M. et al. Decreased colonization of chicks by Salmonella enterica serovar Gallinarum expressing mannose-sensitive FimH adhesin from Salmonella enterica serovar Enteritidis. Vet. Microbiol. 158, 205-210 (2012).

33. Wilson, R. L., Elthon, J., Clegg, S. \& Jones, B. D. Salmonella enterica serovars Gallinarum and Pullorum expressing Salmonella enterica serovar Typhimurium type 1 fimbriae exhibit increased invasiveness for mammalian cells. Infect. Immun. 68, 4782-4785 (2000).

34. Sokurenko, E. V., Vogel, V. \& Thomas, W. E. Catch-bond mechanism of forceenhanced adhesion: counterintuitive, elusive, but widespread? Cell Host Microbe 4, 314-323 (2008)

35. Aprikian, P. et al. Interdomain interaction in the FimH adhesin of Escherichia coli regulates the affinity to mannose. J. Biol. Chem. 282, 23437-23446 (2007)

36. Grimont, P. A. D. \& Weill, F.-X. Antigenic Formulae of the Salmonella Serovars 9th edn (WHO Collaborating Center for Reference and Research on Salmonella, 2007).

37. Liu, F. et al. Subtyping Salmonella enterica serovar enteritidis isolates from different sources by using sequence typing based on virulence genes and clustered regularly interspaced short palindromic repeats (CRISPRs). Appl. Environ. Microbiol. 77, 4520-4526 (2011).

38. Liu, F. et al. Novel virulence gene and clustered regularly interspaced short palindromic repeat (CRISPR) multilocus sequence typing scheme for subtyping of the major serovars of Salmonella enterica subsp. enterica. Appl. Environ. Microbiol. 77, 1946-1956 (2011).

39. Dimarzio, M., Shariat, N., Kariyawasam, S., Barrangou, R. \& Dudley, E. G. Antibiotic resistance in Salmonella Typhimurium associates with CRISPR sequence type. Antimicrob. Agents Chemother. 57, 4282-4289 (2013).

40. Shariat, N. et al. Subtyping of Salmonella enterica serovar Newport outbreak isolates by CRISPR-MVLST and determination of the relationship between CRISPR-MVLST and PFGE results. J. Clin. Microbiol. 51, 2328-2336 (2013).

41. Shariat, N. et al. The combination of CRISPR-MVLST and PFGE provides increased discriminatory power for differentiating human clinical isolates of Salmonella enterica subsp. enterica serovar Enteritidis. Food Microbiol. 34, 164-173 (2013).

42. Darling, A. E., Mau, B. \& Perna, N. T. Progressive Mauve: multiple genome alignment with gene gain, loss and rearrangement. PLoS ONE 5, el1147 (2010).

43. Overbeek, R. et al. The SEED and the Rapid Annotation of microbial genomes using Subsystems Technology (RAST). Nucleic Acids Res. 42, D206-D214 (2014)

44. Zhou, Y., Liang, Y., Lynch, K. H., Dennis, J. J. \& Wishart, D. S. PHAST: a fast phage search tool. Nucleic Acids Res. 39, W347-W352 (2011).

45. Angiuoli, S. V. et al. CloVR: a virtual machine for automated and portable sequence analysis from the desktop using cloud computing. $B M C$ Bioinformatics 12, 356 (2011).

46. Song, J., Xu, Y., White, S., Miller, K. W. \& Wolinsky, M. SNPsFinder--a webbased application for genome-wide discovery of single nucleotide polymorphisms in microbial genomes. Bioinformatics 21, 2083-2084 (2005).

47. Librado, P. \& Rozas, J. DnaSP v5: a software for comprehensive analysis of DNA polymorphism data. Bioinformatics 25, 1451-1452 (2009).

48. Borowsky, R. L. Estimating nucleotide diversity from random amplified polymorphic DNA and amplified fragment length polymorphism data. Mol. Phylogenet. Evol. 18, 143-148 (2001).

49. Delport, W., Poon, A. F., Frost, S. D. \& Kosakovsky Pond, S. L. Datamonkey 2010: a suite of phylogenetic analysis tools for evolutionary biology. Bioinformatics 26, 2455-2457 (2010).

50. Jolley, K. A., Feil, E. J. \& Chan, M. S. Maiden MC. Sequence type analysis and recombinational tests (START). Bioinformatics 17, 1230-1231 (2001). 
51. Carrico, J. A. et al. Illustration of a common framework for relating multiple typing methods by application to macrolide-resistant Streptococcus pyogenes. J. Clin. Microbiol. 44, 2524-2532 (2006).

52. Roy, A., Kucukural, A. \& Zhang, Y. I-TASSER: a unified platform for automated protein structure and function prediction. Nat. Protoc. 5, 725-738 (2010).

53. Wu, S. \& Zhang, Y. LOMETS: a local meta-threading-server for protein structure prediction. Nucleic Acids Res. 35, 3375-3382 (2007).

54. Yang, J., Roy, A. \& Zhang, Y. Protein-ligand binding site recognition using complementary binding-specific substructure comparison and sequence profile alignment. Bioinformatics 29, 2588-2595 (2013).

55. Humphrey, W., Dalke, A. \& Schulten, K. VMD: visual molecular dynamics. J. Mol. Graph. 14, 33-38 27-38 (1996).

56. Blomfield, I. C., McClain, M. S. \& Eisenstein, B. I. Type 1 fimbriae mutants of Escherichia coli K12: characterization of recognized afimbriate strains and construction of new fim deletion mutants. Mol. Microbiol. 5, 1439-1445 (1991).

57. Rhoads, J. M. et al. L-glutamine and L-asparagine stimulate $\mathrm{Na}+-\mathrm{H}+$ exchange in porcine jejunal enterocytes. Am. J. Physiol. 266, G828-G838 (1994).

58. Gonzalez-Vallina, R. et al. Lipoprotein and apolipoprotein secretion by a newborn piglet intestinal cell line (IPEC-1). Am. J. Physiol. 271, G249-G259 (1996).

59. Rusu, D., Loret, S., Peulen, O., Mainil, J. \& Dandrifosse, G. Immunochemical biomolecular and biochemical characterization of bovine epithelial intestinal primocultures. BMC Cell Biol. 6, 42 (2005).

60. Loret, S. et al. Preliminary characterization of jejunocyte and colonocyte cell lines isolated by enzymatic digestion from adult and young cattle. Res. Vet. Sci. 87, 123-132 (2009).

61. Perreault, N. \& Beaulieu, J. F. Primary cultures of fully differentiated and pure human intestinal epithelial cells. Exp. Cell Res. 245, 34-42 (1998).

\section{Acknowledgements}

The study makes use of data generated by the DECIPHER Consortium. A full list of centres that contributed to the generation of the data is available from http://decipher.sanger.ac.uk and via email from decipher@sanger.ac.uk. We thank all the contributors for sharing their Salmonella genomic data, Jason P. Folster and Jean Whichard, Division of Foodborne, Waterborne and Environmental Diseases, Centers for Disease Control and Prevention, Atlanta, GA, USA, for strains, Jeff Washeleski,
Pennsylvania Department of Health, for technical help, and Suzanne Loret, Ernst Heinen and Guy Dandrifosse, University of Liège, Belgium, for their help with bovine cell cultures. We appreciate the critical reading and helpful comments on the manuscript made by Leslie King. This work was funded by NIH grant AI098041, USDA grant 2013 67015-21285 and funds from the University of Pennsylvania Veterinary Center for Infectious Disease and the Center for Host-Microbial Interactions to D.M.S.; X.H., C.Z., X.M. and J.Z. were supported by the China Scholarship Council (CSC).

\section{Author contributions}

D.M.S. and M.Y. designed the experiments, analysed the data and wrote the manuscript; M.Y., X.H., L.D.M., C.Z., X.M., J.Z. and R.W. performed the experiments; and R.S., R.S.K., G.P.F., S.Z., P.F.M., F.-X.W., J.G.M., C.A., W.F.F., R.A.E., D.B., N.R.Z. and S.C.R. assisted with the design, performance and analysis of various experiments.

\section{Additional information}

Accession codes: All the sequence data produced in this study were submitted to GenBank under accession number PRJNA297164.

Supplementary Information accompanies this paper at http://www.nature.com/ naturecommunications

Competing financial interests: The authors declare no competing financial interests.

Reprints and permission information is available online at http://npg.nature.com/ reprintsandpermissions/

How to cite this article: Yue, M. et al. Allelic variation contributes to bacterial host specificity. Nat. Commun. 6:8754 doi: 10.1038/ncomms9754 (2015).

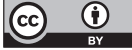

This work is licensed under a Creative Commons Attribution 4.0 International License. The images or other third party material in this article are included in the article's Creative Commons license, unless indicated otherwise in the credit line; if the material is not included under the Creative Commons license, users will need to obtain permission from the license holder to reproduce the material. To view a copy of this license, visit http://creativecommons.org/licenses/by/4.0/ 


\section{Corrigendum: Allelic variation contributes to bacterial host specificity}

Min Yue, Xiangan Han, Leon De Masi, Chunhong Zhu, Xun Ma, Junjie Zhang, Renwei Wu, Robert Schmieder,

Radhey S. Kaushik, George P. Fraser, Shaohua Zhao, Patrick F. McDermott, François-Xavier Weill, Jacques G. Mainil, Cesar Arze, W. Florian Fricke, Robert A. Edwards, Dustin Brisson, Nancy R. Zhang, Shelley C. Rankin \& Dieter M. Schifferli

Nature Communications 6:8754 doi: 10.1038/ncomms9754 (2015); Published 30 Oct 2015; Updated 8 Aug 2017

In Fig. 3 of this Article, the numbers of isolates studied for the fimH41_Newp, fimH44_Newp and fimH45_Newp alleles were inadvertently swapped. The correct version of this figure appears below as Fig. 1.

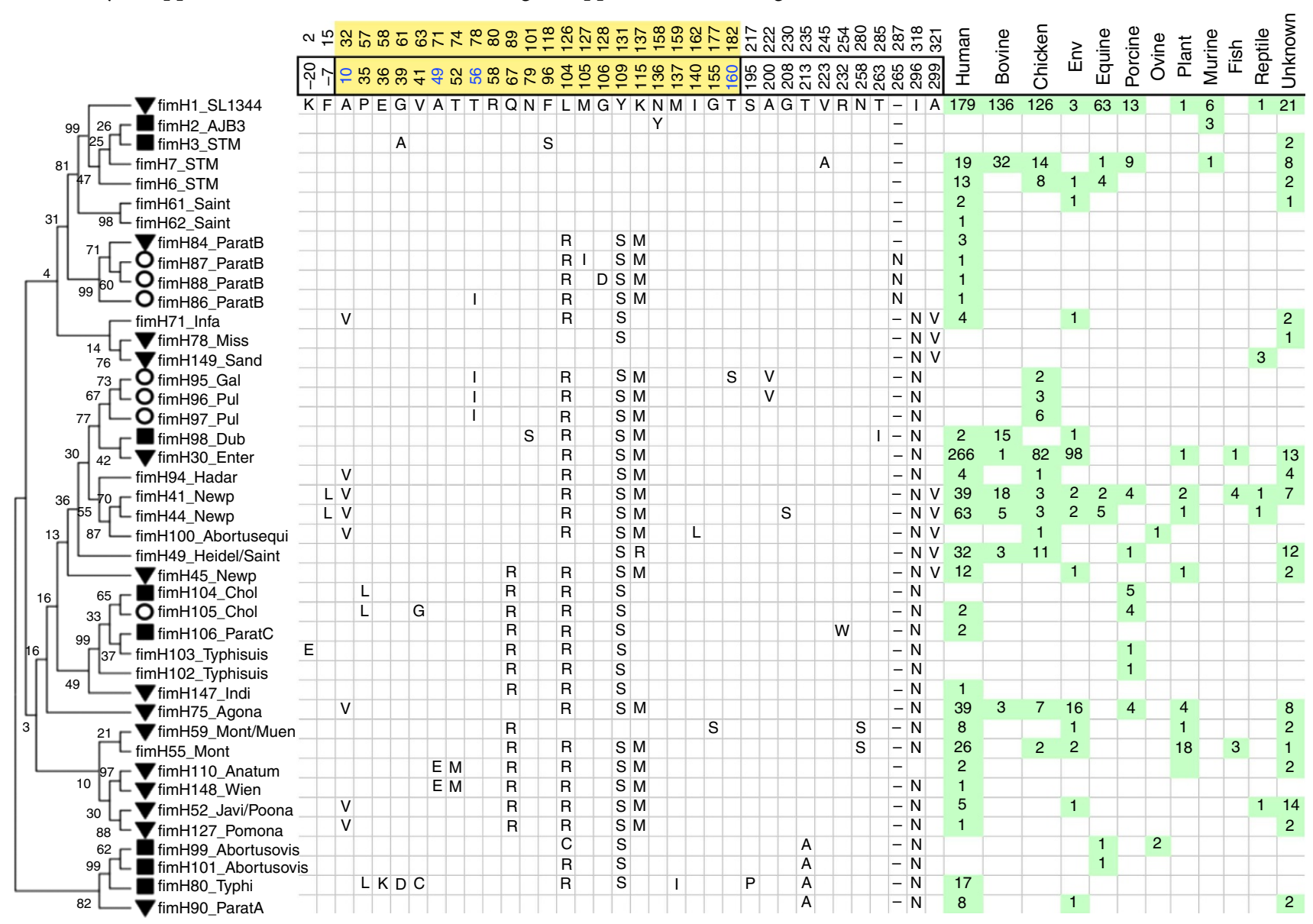

Figure 1

(c) This work is licensed under a Creative Commons Attribution 4.0 International License. The images or other third party material in this article are included in the
article's Creative Commons license, unless indicated otherwise in the credit line; if the material is not included under the Creative Commons license, users will need to obtain permission from the license holder to reproduce the material. To view a copy of this license, visit http://creativecommons.org/licenses/by/4.0/

(C) The Author(s) 2017 